\title{
Changes in Bone Mineral Density in Women Following 1-year Gastric Bypass Surgery, Published by Casagrande DS et al.- Reply
}

\author{
Daniela Schaan Casagrande • Rodolfo Schneider • \\ Beatriz D. Schaan
}

We would like to address some of the questions that Dr. Pluskiewicz et al. [1] raises in his recent letter. Bone mineral density (BMD) measurements are affected by precision and accuracy errors. Precision errors, usually evaluated in terms of the coefficient of variation $(\mathrm{CV})$, measure the reproducibility of BMD results in individual patients and can be demonstrated by performing repeated scans on a representative sample. The BMD changes observed on follow-up scans can be interpreted as the least significant change (LSC), equal to approximately three times the CV. Changes greater than the LSC would be regarded as clinically significant $[2,3]$.

In the São Lucas Hospital of Pontificia Universidade Católica do Rio Grande do Sul (HSL PUCRS) where the dual-energy X-

\footnotetext{
D. S. Casagrande $(\bowtie)$

Obesity and Metabolic Syndrome Center of Hospital São Lucas, Pontificia Universidade Católica do Rio Grande do Sul (COM HSL-PUCRS), Av. Ipiranga 6690, Conjunto 309, 90610-000 Porto Alegre, Brazil

e-mail: casagrandedaniela@gmail.com

R. Schneider

Postgraduate Program in Biomedical Gerontology, Pontifícia Universidade Católica do Rio Grande do Sul, Porto Alegre, Brazil

\section{S. Casagrande $\cdot$ B. D. Schaan}

Postgraduate Program in Medical Sciences: Endocrinology and Metabolism, Universidade Federal do Rio Grande do Sul, Hospital de Clinicas Porto Alegre, Porto Alegre, Brazil

B. D. Schaan

Department of Internal Medicine, Faculty of Medicine,

Universidade Federal do Rio Grande do Sul, Porto Alegre, Brazil
}

ray absorptiometry exams were made, the $\mathrm{CV}$ is $2 \%$ in the lumbar spine and $3 \%$ in the proximal femur (femoral neck and total femur), and the LSC is $5.6 \%$ for the lumbar spine and $8.5 \%$ for the proximal femur. Applying this method of evaluation to our data means that BMD results for women reported in our study [4] decreased 7.3, 8.8, and 8.6\% in the lumbar spine, femoral neck, and total femur, respectively, after gastric bypass surgery. We have not reported our results using the LSC, as this index was questioned due to changes associated with the patient's BMI [3]. Such considerations imply that clinically significant differences between successive BMD results obtained from smaller patients may be unidentified and changes in larger patients may be inappropriately identified as valid [3].

As BMD results depend on multiple factors other than those determined by bariatric surgery, it is clear that not in all subjects after the procedure we should expect significant bone changes. Moreover, Pluskiewicz et al. [1] reported follow up data for only 6 months, which may explain their inability to uncover these changes as we did through a 1year follow-up period.

\section{References}

1. Pluskiewicz W, Buzga $\mathrm{M}$, et al. Bone mineral changes in spine and proximal femur in individual obese women after laparoscopic sleeve gastrectomy: a short-term study. Obes Surg. 2012;22(7):1068-76.

2. Blake GM, Fogelman I. An update on dual-energy X-ray absorptiometry. Semin Nucl Med. 2010;40(1):62-73.

3. Nelson L, Gulenchyn KY, et al. Is a fixed value for the least significant change appropriate? J Clin Densitom. 2010;13(1):18-23.

4. Casagrande DS, Repetto G, et al. Changes in bone mineral density in women following 1-year gastric bypass surgery. Obes Surg. 2012;22(8):1287-92. 